\title{
Cognitive dysfunction in mice underwent cardiac arrest and cardiopulmonary resuscitation evaluated by contextual fear-conditioning test
}

Ye Ma, Chan Chen, Ming Li, Qiao Wang, Bin Liu, Hai Yu

Department of Anaesthesiology, West China Hospital, Sichuan University, Chengdu, China

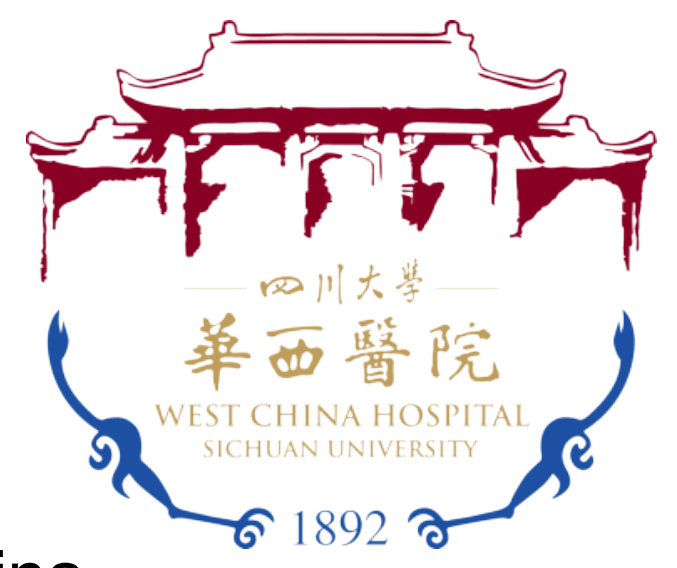

\section{BACKGROUND}

Whole-body ischemia-reperfusion injury (IRI) induced by cardiac arrest (CA) and cardiopulmonary resuscitation (CPR) may lead to severe post-resuscitation organ dysfunction, especially neurological disorder. Cognitive dysfunction has been reported in patients experienced CA and CPR process. Fear conditioning test has been used as a valuable test in several studies to access learning ability and memory in rodents. However, the significance of fear conditioning test to evaluate cognitive disorder in mice underwent CA and CPR has never been reported. Therefore, we investigated whether cognitive dysfunction in mice underwent CA and CPR could be confirmed through fear conditioning test.

\section{METHODS}

C57BL/6 male mice weighted 20-25g were randomly assigned to 2 groups: sham and $\mathrm{CA} / \mathrm{CPR}$ group. $\mathrm{CA}$ was induced by injection of $0.08 \mathrm{mg} / \mathrm{g}$ potassium chloride through PE-10 catheter inserted into jugular vein. CPR was initiated 5 minutes after CA with the frequency of 300-400 beat per minute. At the same time, $0.4 \mu \mathrm{g} / \mathrm{g}$ epinephrine was given slowly. Return of spontaneous circulation (ROSC) was confirmed from electrocardiogram and visual heart beat . Mice were trained with conditional stimulus (cue tone) and aversive, unconditional stimulus (foot shock) the day before modeling in fear conditioning chamber. Fear conditioning test was conducted on the second day after modeling (Figure 1). Freezing behavior showed a better cognitive function when they were re-exposed in the conditional stimulus. We record the survival rate, freezing scores of mice.

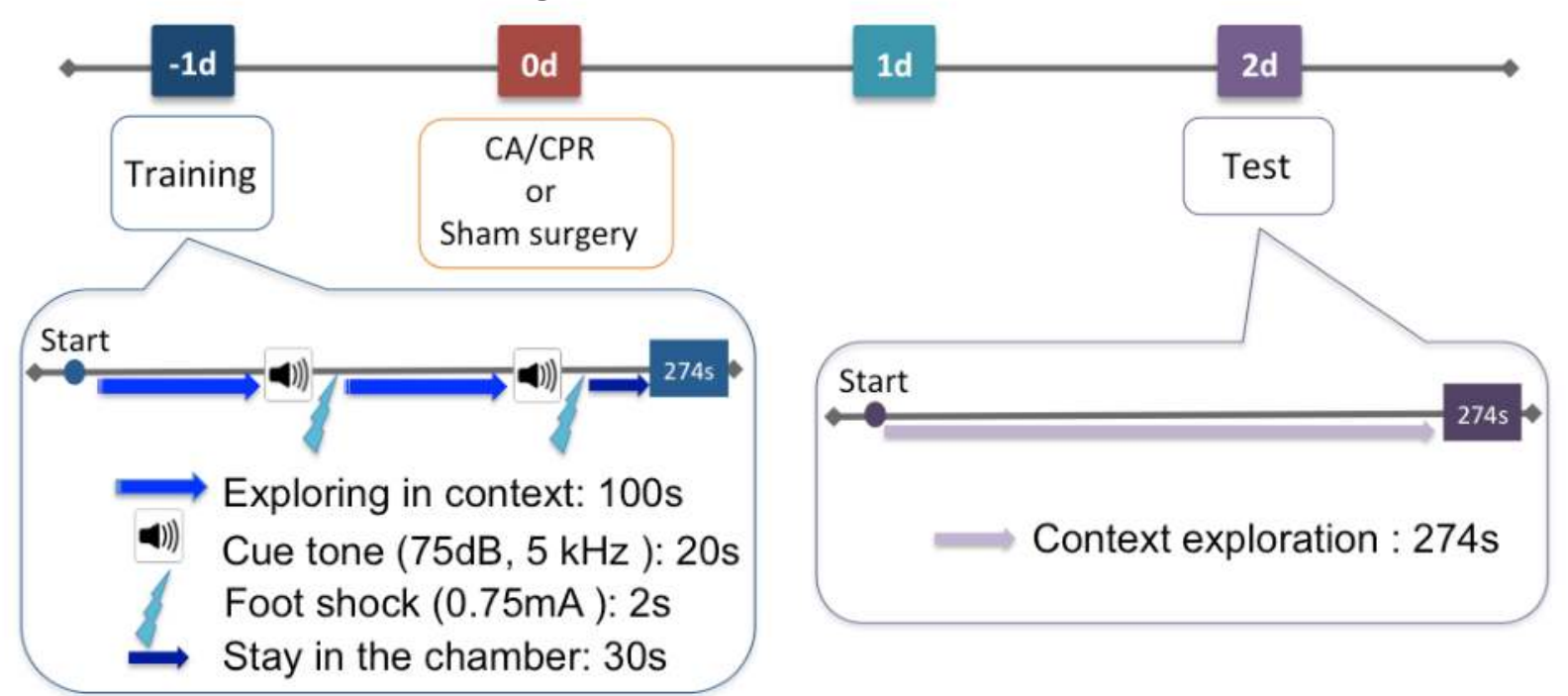

Figure 1. Schematic outline of contextual fear-conditioning test. Protocol in the present study was based on the study of Tterrando and colleagues. Training was conducted the day before $\mathrm{CA} / \mathrm{CPR}$ performance and test was conducted on day 3 after training.

\section{RESULTS}

Survival rate on day 3 after modeling was $46.7 \%$ in CA/CPR group $(n=15)$ and $100 \%$ in sham group $(n=15)$. Percentage of freezing time was significant lower in CA/CPR group $(n=6)$ compared with sham group $(\mathrm{n}=6)(15.04 \pm 2.323$ vs. $50.95 \pm$ 1.830) (Figure $2 \mathrm{~A}, * * * * P<0.0001$ ), indicating a higher cognitive disorder with $\mathrm{CA} / \mathrm{CPR}$ process.

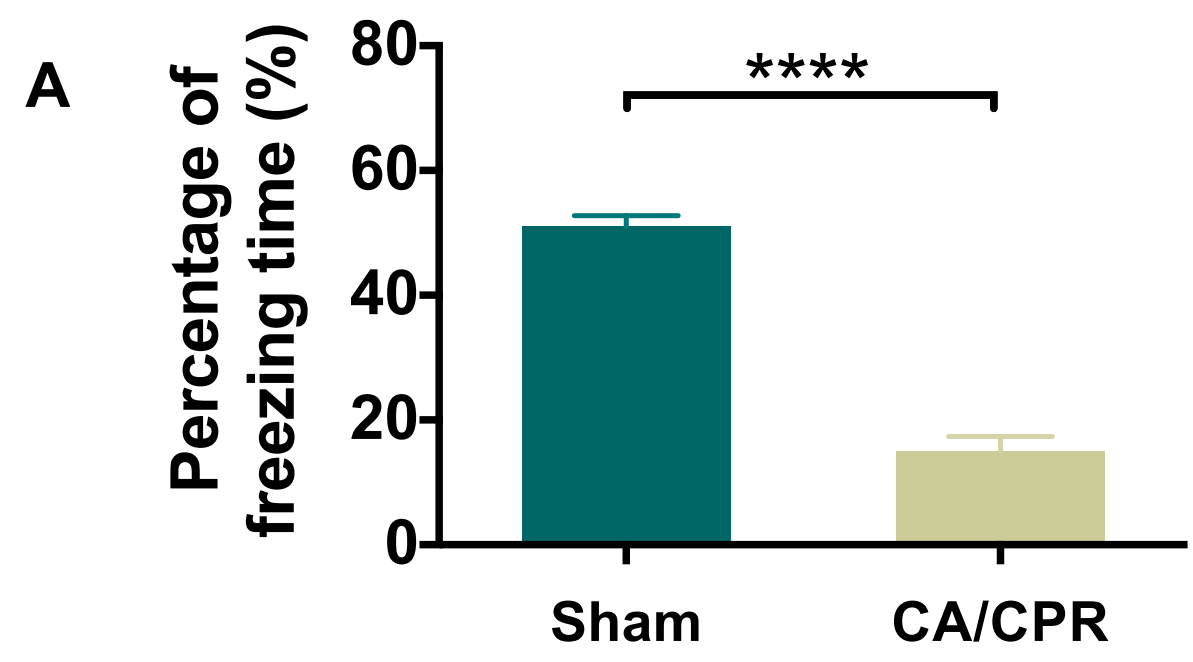

B
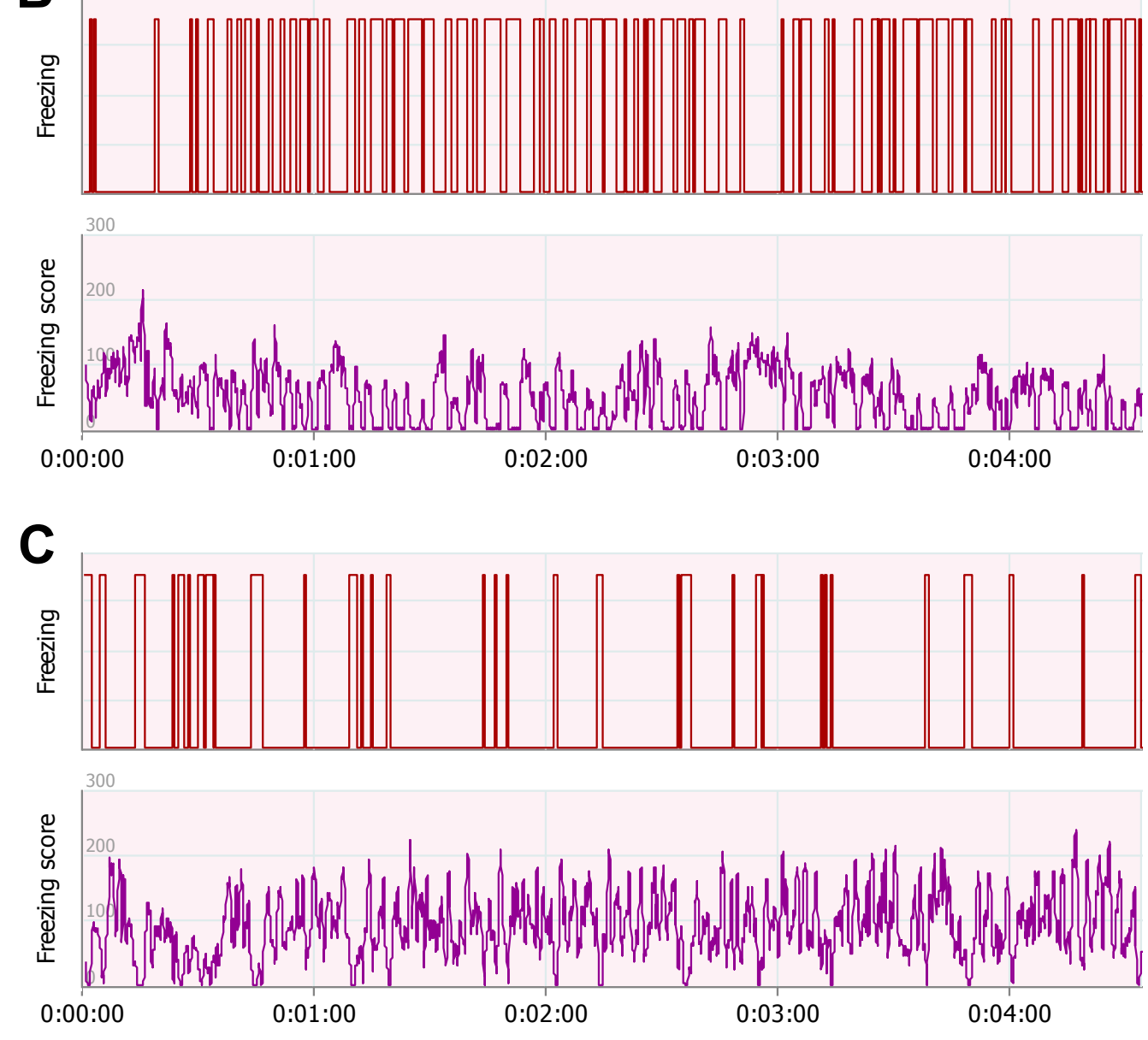

Figure 2. Percentage of freezing time in contextual fearconditioning test and the representative recording graph. A. Percentage of freezing time of two groups in fear-conditioning test. Percentage of freezing time was defined as the radio of freezing time to total time of test process. Higher value indicated better memory and learning ability. Data are presented as mean \pm SEM. $* * * * P<0.0001$. $\mathrm{CA} / \mathrm{CPR}$, cardiac arrest and cardiopulmonary resuscitation. B. Representative recording graph of freezing condition and freezing scores of mice in two groups.

\section{CONCLUSIONS}

This study demonstrated that mice could have cognitive disorder after CA/CPR. Also, fear conditioning is a valuable test of cognitive dysfunction after $\mathrm{CA} / \mathrm{CPR}$ process in mice. 